INPLASY

PROTOCOL

To cite: Xiong et al. Effect of immersive technology on $\mathrm{K}-12$ and higher education students' creativity: A systematic review and meta-analysis. Inplasy protocol 202210108. doi: 10.37766/inplasy2022.1.0108

Received: 21 January 2022

Published: 21 January 2022

Corresponding author: Juan Xiong

xiongjuan0@sina.com

Author Affiliation:

Chongqing university.

Support:

Project:2020CDJSK07XK14.

Review Stage at time of this submission: Completed but not published.

Conflicts of interest: None declared.

\section{Effect of immersive technology on K-12 and higher education students' creativity: A systematic review and meta-analysis}

Xiong, J1; Wen, J2; Zhang, S3; Pei, G4; Han, X55.

Review question / Objective: (1) What are research findings on the relation between immersive technology and creativity in higher education? (2) Is immersive technology conductive to improving students' creativity? (3) To what extent does immersive technology affect students' creativity? (4) Are there significant differences in moderating variables such as type of intervention, subject, grade level, time, and team or individual? (5) What are future research directions regarding the educational use of immersive technology based on the reviewed literature?

Condition being studied: Creativity is recognized as a crucial 21st-century skill, and immersive technology can stimulate students' creativity.

INPLASY registration number: This protocol was registered with the International Platform of Registered Systematic Review and Meta-Analysis Protocols (INPLASY) on 21 January 2022 and was last updated on 21 January 2022 (registration number INPLASY202210108).

\section{INTRODUCTION}

Review question / Objective: (1) What are research findings on the relation between immersive technology and creativity in higher education? (2) Is immersive technology conductive to improving students' creativity? (3) To what extent does immersive technology affect students' creativity? (4) Are there significant differences in moderating variables such as type of intervention, subject, grade level, time, and team or individual? (5) What are future research directions regarding the educational use of immersive technology based on the reviewed literature?

Condition being studied: Creativity is recognized as a crucial 21 st-century skill, 
and immersive technology can stimulate students' creativity.

\section{METHODS}

Search strategy: An initial search was carried out in May 2021 using a Boolean search phrase to combine the search terms and identify relevant research outputs. We searched for any relevant cross-sectional studies on immersive technology, and we searched Web of Science (Multidisciplinary Sciences, Education, Educational Research, Education Scientific Disciplines, Educational Psychology) and EBSCO Host (British Education Index, British Education Abstracts, ERIC, PsycINFO, and Teacher Reference Center) for articles published in English. Additionally, we searched for articles that focused on the topic of this systematic review in the following selected journals: "Review of Educational Research," "Educational Psychologist," "Computers \& Education," "Educational Research Review," "Internet and Higher Education," "Review of Research in Education," "Computers in Human Behavior," "Virtual Reality," "Advanced Engineering Informatics," "Journal of Creative Behavior," "Psychology of Aesthetics," and "Creativity, and the Arts" (Appendix A). We search generated 3016 items. After the removal of duplicates and application of eligibility criteria, 18 articles were selected for meta-analysis.

Participant or population: K-12 and higher education students.

Intervention: Immersive technology.

Comparator: Traditional teaching.

Study designs to be included: We conducted this meta-analysis following the Preferred Reporting Items for Systematic Reviews and Meta-Analyses (PRISMA) standards of quality for reporting metaanalysis.

Eligibility criteria: Two trained coders coded each phase of the current metaanalysis to reduce concerns with researcher subjectivity. Data selection is critical to conducting a meta-analysis, and thus, this study employed a systematic process (see Fig. 1). The five inclusion criteria were as follows: (1) the articles analysed the influence of immersive technology on students' creativity; (2) they were group-designed randomized controlled trials that compared the effects of immersive technology-based teaching interventions to control conditions; (3) they targeted students rather than the general population or adult learners; (4) they included statistical information such as one correlation or indicators that can be converted into an effect index; and (5) they were not observational reports, dissertations, unpublished manuscripts, conference abstracts, or intervention studies that did not directly evaluate students' creativity, and newsletter articles.

Information sources: Electronic databases.

Main outcome(s): Students' creativity.

Quality assessment / Risk of bias analysis: Quality assessment was performed based on the literature quality evaluation method of Valentine \& Cooper.

Strategy of data synthesis: The effect size calculations were based on between-group comparisons of pre-post change scores using pooled pretest standard deviations. To evaluate the efficacy of the interventions, we calculated Hedges's g (Hedges, 1981) and 95\% confidence intervals (Cls).

Subgroup analysis: Type of intervention, subject, grade, time and individual or team.

Sensitivity analysis: Figure 3 presents a funnel plot of precision by Hedges's $g$ relating pooled effect sizes on the main outcomes of the studies to the standard errors (SEs) of the estimates. According to Figure 3, the 18 independent research data points were basically distributed at the centre of the combined effect size. This indicates that the papers included had a low possibility of publication bias, and this study had a certain credibility. However, some points are in a free state, so there 
may be heterogeneity between the included studies. This conclusion is consistent with the above heterogeneity test results.

Language: English.

Country(ies) involved: China.

Keywords: immersive technology; students' creativity; meta-analysis

Contributions of each author:

Author 1 - Juan Xiong - Conceptualization, Methodology, Formal analysis, Data coding, Data analysis, Writing-method and results sections, Writing-original draft, Visualization, Supervision.

Email: xiongjuan0@sina.com

Author 2 - Jianlin Wen - Conceptualization, Methodology, Formal analysis, Data coding, Data analysis, Visualization, Funding acquisition.

Email: forrestwin2022@163.com

Author 3 - Songrui Zhang - Data coding, Data analysis, Participation in discussion and writing.

Email: 15295775517@163.com

Author 4 - Guangshu Pei - Data coding, Data analysis, Participation in discussion.

Email: pgs@cqu.edu.cn

Author 5 - Xu Han - Data coding, Data analysis.

Email: 96hxanu@gmail.com 